\title{
Vestibular Disorders: Pearls and Pitfalls
}

\author{
Woo Young Choi, MD, PhD, FRCPC ${ }^{1}$ Daniel R. Gold, DO \\ ${ }^{1}$ Department of Neurology, The Johns Hopkins University School of \\ Medicine, Baltimore, Maryland \\ 2 Departments of Neurology, Ophthalmology, Neurosurgery, \\ Otolaryngology - Head and Neck Surgery, Emergency Medicine, \\ The Johns Hopkins University School of Medicine, Baltimore, \\ Maryland \\ Address for correspondence Daniel R. Gold, DO, Departments of \\ Neurology, Ophthalmology, Neurosurgery, Otolaryngology-Head \\ and Neck Surgery, Emergency Medicine, The Johns Hopkins University \\ School of Medicine, 600 North Wolfe Street, Pathology 2-210, \\ Baltimore, MD 21287 (e-mail: Dgold7@jhmi.edu).
}

Semin Neurol 2019;39:761-774.
Abstract
Keywords
- vertigo
- dizziness
- acute vestibular syndrome
- vestibular
- HINTS exam

Dizziness and vertigo are symptoms that commonly lead patients to seek neurologic or emergency care. Because symptoms are often vague and imprecise, a systematic approach is essential. By categorizing vestibular disorders based on the timing, triggers, and duration of symptoms, as well as emphasizing focused ocular motor and vestibular examinations, the majority of vestibular diagnoses can be made at the bedside. This paper will discuss the pearls and pitfalls in the history and examination of the most common acute, episodic, and chronic vestibular disorders.

\section{Taking the History}

\section{Background and Definitions}

Common vestibular disorders such as benign paroxysmal positional vertigo (BPPV) and vestibular neuritis are frequently misdiagnosed, ${ }^{1,2}$ and their management tends to be variable and nonevidence based., ${ }^{3,4}$ Correctly diagnosing peripheral vestibular disorders is important because they are common and evidence-based treatments improve outcomes. ${ }^{5-8}$ Also, correctly diagnosing central vestibular disorders, such as strokes and transient ischemic attacks (TIAs), are of paramount importance because posterior circulation strokes commonly present with acute dizziness or vertigo, and the consequences of missing a posterior fossa stroke can be devastating. 9,10

Patients with vestibular conditions typically present with symptoms of vertigo, dizziness, oscillopsia, or unsteadiness. Although the terms and descriptors used by patients are often vague, imprecise and even misleading, ${ }^{11}$ international consensus on the definitions of the symptom types have been published and are described below. ${ }^{12,13}$

Vertigo is defined as a sensation of self-motion or of motion of the external environment when none is occurring, or a distorted sensation of self or external motion during an otherwise normal head movement. Vertigo gen- erally, but not always, indicates a vestibular imbalance caused by processes affecting the semicircular canal (SCC) afferents in the labyrinth, the eighth cranial nerve $(\mathrm{CN})$, or vestibular connections through the brainstem or cerebellum prior to reaching the ocular motor nuclei. Vertigo can be divided into spinning or nonspinning (the latter comprising swaying, tilting, bobbing, bouncing, or sliding), ${ }^{12,14,15}$ and these types may help to distinguish whether symptoms originate in the SCC (anterior, horizontal, and posterior canals) or otolithic (utricle and saccule) pathways, respectively. However, it is important to understand that patients with orthostatic hypotension, cardiac arrhythmias, or other non-vestibular conditions may also experience vertigo. ${ }^{16}$ While a patient's description of their symptoms is of some importance, the description alone lacks specific diagnostic value in most cases.

Dizziness is the sensation of disturbed or impaired spatial orientation without a false or distorted sense of motion. ${ }^{12,14,15}$ Dizziness may be experienced in vestibular or nonvestibular conditions. Oscillopsia is a specific term defined as a false (visual) sense of environmental motion. For simplicity, it can be further divided into "sitting" or "walking" oscillopsia. In "sitting" oscillopsia, the visual world moves independent of head movement-for example, the
Issue Theme Neuro-Ophthalmology; Guest Editor, Sashank Prasad, MD
Copyright (c) 2019 by Thieme Medical Publishers, Inc., 333 Seventh Avenue, New York, NY 10001, USA. Tel: +1(212) 584-4662.
DOI https://doi.org/ $10.1055 / \mathrm{s}-0039-1698752$. ISSN 0271-8235. 
visual symptoms accompanying nystagmus or saccadic intrusions/oscillations. In "walking" oscillopsia, the visual world moves during head movements-for example, oscillopsia while walking that occurs with bilateral vestibular loss (most common) or when vestibular function is preserved but suppression of the vestibular-ocular reflex is impaired (less common). As oscillopsia is a visual sensation, it should abate with the eyes closed. However, closing the eyes will not eliminate vestibular symptoms such as dizziness or vertigo. In some cases, oscillopsia and other vestibular symptoms may both be present; for example, in acute vestibular neuritis, closing the eyes will eliminate the symptom of oscillopsia associated with nystagmus, but the feeling of vertigo will persist. Simply put, oscillopsia is a visual sensation (absent with eyes closed), while vertigo is a balance sensation (still present with eyes closed). Other definitions to be aware of include unsteadiness, which is the feeling of instability while seated, standing, or walking, without a directional preference ${ }^{12,15}$; presyncope, which is the sensation of impending loss of consciousness; and syncope, which is a transient loss of consciousness related to global cerebral hypoperfusion.

\section{Pitfalls: Traditional Approach to Dizziness}

In the traditional approach to dizziness, the most important distinguishing feature was the symptom type. Symptoms were classified according to vertigo (the illusion of spinning or other false motion), presyncope (a feeling of impending faint), disequilibrium (unsteadiness when walking), or nonspecific dizziness (other balance-related sensation not fitting the prior three categories, such as lightheadedness, wooziness, and giddiness). ${ }^{17}$ The advantage of this approach is that it can quickly narrow the differential diagnosis and guide diagnostic testing, with vertigo suggesting vestibular causes, presyncope implying cardiovascular causes, unsteadiness prompting a search for neurologic causes, and nonspecific dizziness inferring psychiatric or metabolic causes. This symptom-type approach has been often frequently endorsed, and frontline physicians consider the type of dizziness to be very important. ${ }^{18}$ However, several studies show that the type of vestibular symptom is not reliably reported by patients and does not validly discriminate among different causes of dizziness. ${ }^{11,19} \mathrm{~A}$ symptom-type approach by itself can easily lead a clinician to pursue the wrong diagnosis with inappropriate diagnostic testing and should be avoided.

Recently, a novel evidence-based approach to taking the vestibular history has been proposed, which emphasizes timing and triggers rather than the patient's qualitative description of the symptom. ${ }^{14,20}$

\section{Pearls: Triage-TiTrATE-Test Method}

Historical elements that should be emphasized when evaluating a patient with vestibular symptoms include the distinction between new or recurrent attacks, symptom onset, triggers, associated symptoms, and duration of spells. ${ }^{14,20}$ The triage-TiTrATE (timing, triggers, and targeted examinations)-test method offers a convenient mnemonic to organize this approach. ${ }^{14}$

\section{Triage (Triage-TiTrATE-Test)}

This step is intended to quickly identify the most dangerous and nonvestibular conditions through history (e.g., chest pain, shortness of breath, symptoms typical of panic attacks, new medications, or recent dose adjustments); vital signs (e.g., hypertensive emergency, hypotension, and bradycardia); and basic, targeted ancillary testing (e.g., EKG, blood glucose level, and anticonvulsant serum levels). ${ }^{21}$ of note, medication side effects are common causes of constant dizziness or vertigo and can be associated with specific ocular motor abnormalities. ${ }^{22}$ For example, anticonvulsant toxicity can cause vestibular symptoms and gaze-evoked nystagmus, and lithium can cause downbeat nystagmus.

\section{Timing (Triage-TiTrATE-Test)}

The following factors are critically important to correctly diagnose a vestibular condition: (1) how did the symptoms begin (i.e., did they evolve over seconds, minutes, hours, days, or more)?; (2) is this an acute and prolonged first event (monophasic), or are the symptoms episodic (with discrete attacks) or chronic (constant for weeks-months)?; and (3) what is the duration of symptoms (especially important for episodic conditions)?

\section{Triggers (Triage-TiTrATE-Test)}

Correctly identifying the triggers of vestibular symptoms can significantly narrow the differential diagnosis. Positional triggers are very common, where symptoms come on immediately after a head movement (e.g., BPPV). In other cases, the symptoms may be time-locked to a head movement (e.g., unilateral or bilateral vestibulo-ocular reflex [VOR] deficit). Notably, vestibular migraine (VM) patients often possess symptoms that are time-locked to head movements (sometimes referred to as head motion intolerance), although the VOR is normal. Symptoms can be visually induced by a complex or busy visual environment in VM, persistent postural perceptual dizziness, and other vestibular conditions that lead to overreliance on visual inputs with resultant "visual vertigo." 23 Triggers can be sound induced (Tullio's phenomenon), ${ }^{24}$ for example, in superior canal dehiscence syndrome (SCDS). ${ }^{25}$ The Valsalva maneuver may induce symptoms and signs (nystagmus) with a cervicomedullary lesion (e.g., Arnold-Chiari) or in SCDS. ${ }^{26}$ An important and common postural cause of dizziness is orthostatic hypotension, which can be historically differentiated from BPPV by the presence of symptoms following rolling over in bed or going from seated to lying in bed in the latter condition. In many cases, there is no discernible trigger, which can also be diagnostically meaningful (e.g., TIA).

\section{Targeted examination (Triage-TiTrATE-Test)}

The proper examination should be designed to test hypotheses raised by the history. For example, the HINTS exam (head impulse, nystagmus, and test of skew) should be performed in the setting of an acute vestibular syndrome (see below), while the Dix-Hallpike (DH) maneuver is performed when BPPV is suspected. 


\section{Test (Triage-TiTrATE-Test)}

Depending on the history and tailored exam, the most appropriate diagnostic testing is ordered.

\section{Box 1: Vestibular History Pitfall}

- Overreliance on qualitative description of symptoms is an ineffective approach to most vestibular disorders and often leads to the wrong diagnosis.

\section{Pearls}

- Once medical and obvious nonvestibular conditions are excluded, the history should focus on symptom timing and the presence or absence of triggers. The diagnosis is usually made at this point (or the differential diagnosis is narrowed substantially), and the targeted exam either confirms the suspected etiology (e.g., DH in BPPV) or allows the clinician to select the appropriate diagnostic test (e.g., MRI and MR angiography in the setting of a HINTS exam suggesting a central cause).

\section{Performing the Examination}

\section{Pitfalls}

A common pitfall to keep in mind is that the clinician may be falsely reassured by a normal general neurological examination of a patient with vestibular symptoms due to a small posterior fossa stroke. ${ }^{27}$ Importantly, dizziness or vertigo is the most frequently presenting symptom in patients with posterior circulation ischemia, ${ }^{28}$ and of those patients presenting with isolated vestibular symptoms due to stroke, approximately $80 \%$ will have a normal general neurologic examination. $^{29}$

While the ocular motor examination is often abnormal in vestibular disorders, ${ }^{29}$ it is frequently underused or misinterpreted by frontline physicians. ${ }^{1,4}$ Many vestibular disorders have characteristic ocular motor findings that allow a specific diagnosis to be made with a high degree of certainty. ${ }^{27}$ For example, posterior canal (PC) BPPV can be confidently diagnosed when symptoms are positionallytriggered, and the DH test elicits the characteristic transient upbeat-torsional nystagmus. Unfortunately, the DH test for PC BPPV is often misused and misinterpreted, ${ }^{16,27}$ despite the fact that it is the gold standard for diagnosis. It is important to know that the DH test can also be misleading when performed in inappropriate circumstances, such as in a patient who has continuous symptoms or spontaneous nystagmus (i.e., both are features inconsistent with BPPV). For example, a patient with a VOR impairment (e.g., vestibular neuritis) or head motion intolerance with a normal VOR (e.g., vestibular migraine) will often experience more intense symptoms (and commonly more intense nystagmus as well) with the DH maneuver. Therefore, performing the $\mathrm{DH}$ in a patient with acute continuous vestibular symptoms will not help to establish the diagnosis (HINTS should be used here), but instead will aggravate the patient's symptoms and may lead to the examiner misinterpreting the $\mathrm{DH}$ as positive for BPPV.

\section{Pearls}

Medical examination maneuvers (e.g., auscultation of the neck and heart, blood pressure, orthostatic measurements) should be performed when appropriate. Given the extensive ocular motor and vestibular pathways within the brainstem and cerebellum, a careful cranial nerve and eye movement examination is critical. The general neurologic examination can be normal in patients presenting with isolated dizziness or vertigo due to small brainstem or cerebellar strokes, but the ocular motor and vestibular examination will almost always provide the clues toward the correct diagnosis ( - Table 1). ${ }^{27}$ Gait and stance are usually the most helpful components of the general neurologic examination in patients with isolated vestibular symptoms, specifically evaluating for truncal or gait ataxia. An otoscopic examination may provide clues regarding the etiology of dizziness, especially when a peripheral infectious etiology is suspected.

\section{Box 2: Vestibular Examination Pitfalls}

- A normal general neurologic examination does not imply that the etiology of vertigo is peripheral; the ocular motor and vestibular bedside examination should be emphasized instead.

- The DH maneuver should be performed and interpreted in the correct setting. It may aggravate symptoms in patients with any vestibular condition, but this should not be falsely interpreted as a positive $\mathrm{DH}$.

\section{Pearls}

- The DH maneuver should be performed in patients with positionally-triggered symptoms.

- Once an appropriate differential diagnosis is established based on the timing and triggers of vestibular symptoms, a targeted ocular motor and vestibular examination will commonly lead to the correct diagnosis (-Table $\mathbf{1}$ ).

\section{Making the Diagnosis}

Based on the timing and triggers of symptoms, vestibular disorders can be categorized as acute, episodic (triggered or spontaneous), or chronic vestibular syndromes.

\section{Acute Vestibular Syndrome}

The acute vestibular syndrome (AVS) consists of rapid onset, continuous vertigo or dizziness lasting for $>24$ hours, with associated, nausea, vomiting, spontaneous nystagmus, 
Table 1 The most common vestibular conditions categorized by timing and triggers, with specific ocular motor and vestibular features that should be sought for each

\begin{tabular}{|c|c|c|}
\hline & $\begin{array}{l}\text { Vestibular conditions } \\
\text { to consider }\end{array}$ & Targeted ocular motor and vestibular examinations \\
\hline $\begin{array}{l}\text { Acute vestibular } \\
\text { syndrome } \\
(>24 \mathrm{~h})\end{array}$ & $\begin{array}{l}\text { 1. Vestibular neuritis } \\
\text { 2. Stroke (demyelination } \\
\text { and other central } \\
\text { etiologies less common) } \\
\text { 3. Wernicke's }\end{array}$ & $\begin{array}{l}\text { 1. HINTS + : HIT abnormal (see example-https://collections.lib.utah.edu/ark:/ } \\
\text { 87278/sx398q2) AND unidirectional nystagmus that obeys Alexander's law } \\
\text { (see example-https://collections.lib.utah.edu/ark:/87278/s64205qx) AND } \\
\text { skew deviation absent (see alternate cover testing at } 1 \text { and } 3 \text { minutes-https:// } \\
\text { collections.lib.utah.edu/ark:/87278/s6tn1htv) AND no acute hearing loss; } \\
\text { peripheral pattern of HSN } \\
\text { 2. HINTS + : HIT normal (see demonstration in a normal patient-https://collec- } \\
\text { tions.lib.utah.edu/ark:/87278/s63b97tz) OR gaze-evoked nystagmus (see } \\
\text { example-https://collections.lib.utah.edu/ark:/87278/s6kh4n5k) OR skew } \\
\text { deviation present (see example-https://collections.lib.utah.edu/ark:/87278/ } \\
\text { s6c0045t) OR acute hearing loss; look for central patterns of HSN (see example- } \\
\text { https://collections.lib.utah.edu/ark:/87278/s61c5vkg) } \\
\text { 3. Bilaterally abnormal HIT, spontaneous vertical (see example-https://collec- } \\
\text { tions.lib.utah.edu/ark:/87278/s6h74j6d) and gaze-evoked nystagmus are } \\
\text { common, also 6th NP, ataxia }\end{array}$ \\
\hline $\begin{array}{l}\text { Episodic } \\
\text { spontaneous } \\
\text { vestibular } \\
\text { syndrome }^{\mathrm{a}}\end{array}$ & $\begin{array}{l}\text { 1. TIA } \\
\text { 2. Vestibular migraine } \\
\text { 3. Meniere's } \\
\text { 4. Vestibular paroxysmia }\end{array}$ & $\begin{array}{l}\text { 1. Usually symptoms have resolved and eye movement exam is normal; otherwise, } \\
\text { may use HINTSb } \\
\text { 2. Can see peripheral or central patterns of nystagmus (spontaneous, gaze-evoked, } \\
\text { head shaking-induced, positional) during the attack, often normal interictally; } \\
\text { often spontaneous, but typical migraine triggers are common too } \\
\text { 3. Nystagmus can be in excitatory or inhibitory patterns during the attack, often } \\
\text { normal interictally } \\
\text { 4. Hyperventilation-induced nystagmus (see demonstration in a normal patient- } \\
\text { https://collections.lib.utah.edu/ark:/87278/s6pz98ht) }\end{array}$ \\
\hline $\begin{array}{l}\text { Episodic } \\
\text { triggered } \\
\text { vestibular } \\
\text { syndrome }\end{array}$ & $\begin{array}{l}\text { 1. BPPV } \\
\text { 2. Central positional } \\
\text { vertigo or nystagmus } \\
\text { 3. SCDS }\end{array}$ & $\begin{array}{l}\text { 1. Dix-Hallpike in posterior canal variant (see example-https://collections.lib. } \\
\text { utah.edu/ark:/87278/s6s79d1w) and supine roll test in horizontal canal variant } \\
\text { (with bow and lean to localize, see example-https://collections.lib.utah. } \\
\text { edu/ark:/87278/s68h2wk9) } \\
\text { 2. Consider this diagnosis when history/exam features are atypical for BPPV, } \\
\text { including an unexpected nystagmus vector; downbeat (see video example } \\
\text { https://collections.lib.utah.edu/ark:/87278/s66t3w9k) and apogeotropic } \\
\text { nystagmus patterns are most common } \\
\text { 3. Valsalva and pinched-nose Valsalva (see example-https://collections.lib.utah.- } \\
\text { edu/details?id=1213443), tragal compression, loud sounds }\end{array}$ \\
\hline $\begin{array}{l}\text { Chronic } \\
\text { vestibular } \\
\text { syndrome }\end{array}$ & $\begin{array}{l}\text { 1. BVL } \\
\text { 2. PPPD } \\
\text { 3. Cerebellar disease } \\
\text { 4. Acoustic neuroma } \\
\text { 5. Oculopalatal tremor }\end{array}$ & $\begin{array}{l}\text { 1. Bilaterally abnormal HIT (see example-https://collections.lib.utah.edu/ark:/ } \\
\text { 87278/s62z4z8g), additional cerebellar signs can narrow differential and loss of } \\
\text { four or more lines with dynamic visual acuity (see example of how to perform } \\
\text { this maneuver-https://collections.lib.utah.edu/ark:/87278/s6tn19w8) } \\
\text { 2. No typical ocular motor/vestibular findings } \\
\text { 3. Flocculus/paraflocculus: gaze-evoked nystagmus, spontaneous downbeat } \\
\text { nystagmus, saccadic pursuit and VORS (when VOR is present), saccadic } \\
\text { dysmetria (see example-https://collections.lib.utah.edu/ark:/87278/ } \\
\text { s6dj8q9h), alternating skew deviation (see example-https://collections.lib. } \\
\text { utah.edu/ark:/87278/s6d83n91) while additional vestibular loss can narrow } \\
\text { differential (e.g., CANVAS, see example-https://collections.lib.utah.edu/ark:/ } \\
\text { 87278/s6s50fth) } \\
\text { Nodulus/uvula: periodic alternating nystagmus (see example-https://collec- } \\
\text { tions.lib.utah.edu/ark:/87278/s62k013r) } \\
\text { 4. Hyperventilation-induced nystagmus (see example-https://collections.lib. } \\
\text { utah.edu/ark:/87278/s63f8cgs), and Brun's nystagmus (see example-https:// } \\
\text { collections.lib.utah.edu/ark:/87278/s60p4p3j) } \\
\text { 5. Vertical or vertical-torsional pendular nystagmus with palatal tremor } \\
\text { (see example-https://collections.lib.utah.edu/ark:/87278/s6mh1mnm) }\end{array}$ \\
\hline
\end{tabular}

Abbreviations: BPPV, benign paroxysmal positional vertigo; BVL, bilateral vestibular loss; CANVAS, cerebellar ataxia, neuropathy, vestibular areflexia syndrome; HIT, head impulse test; HINTS + , head impulse, nystagmus, test of skew, "Plus" bedside assessment of auditory function; NP, nerve palsy; PPPD, persistent postural perceptual dizziness; SCDS, superior canal dehiscence syndrome.

alf first attack of TIA, vestibular migraine or Meniere's, may be better described as the acute transient vestibular syndrome $(<24$ hours).

bHINTS has been studied in the acute vestibular syndrome, and should not be relied upon in the episodic or acute transient vestibular syndrome. Source: Neuro-Ophthalmology Virtual Education Library: NOVEL. (online) Available from: https://collections.lib.utah.edu/ark:/87278/s68d47vr. 
imbalance, and head motion intolerance. Although vestibular migraine (VM) may also be included in this category, particularly if it is the initial attack, this discussion will be limited to the acute vestibular syndrome, which is generally due to either vestibular neuritis $(V N)$ or stroke/transient ischemic attack (TIA). Furthermore, while VM and TIA are episodic conditions, $\mathrm{VN}$ and stroke are usually monophasic.

HINTS is a three-step bedside ocular motor examination that has a sensitivity higher than even MR with diffusionweighted imaging (DWI) to detect an acute stroke in the AVS. This is especially due to the fact that MR-DWI may be falsely negative approximately $20 \%$ of the time in the first 24 to 48 hours. ${ }^{29,30}$ A "peripheral" HINTS exam should include the following: abnormal (ipsilesional) head impulse test (HIT) AND unidirectional (contralesional) nystagmus AND negative test of skew. For example, right vestibular neuritis causes an abnormal HIT to the right, unidirectional mixed horizontal-torsional left-beating nystagmus (LBN) (that follows Alexander's law, with increased LBN when looking to the left and decreased LBN or no nystagmus in right gaze), and no vertical refixation movement of the eyes with tests of ocular alignment. The HINTS exam should be considered "central" if any of the following are present: normal HIT OR bidirectional or direction-changing gaze-evoked nystagmus OR presence of a skew deviation. Commonly, one or two of the three steps will appear "peripheral," but the presence of even one "central" sign should point the examiner toward a dangerous etiology that requires expeditious neuroimaging.

It can become particularly confusing when patients present with combined peripheral (e.g., abnormal HIT) and central vestibular features (e.g., direction-changing nystagmus). This is often due to (1) anterior inferior cerebellar artery (AICA) territory strokes, causing simultaneous brainstem/cerebellar, and labyrinthine ischemia; or (2) solitary lesions involving the vestibular nucleus, as the peripheral SCC and otolithic afferents become central and converge on the vestibular nuclei. In addition to HINTS, impaired (or asymmetric) smooth pursuit and/or saccade dysmetria can have further localizing value in this situation. Characteristic findings have been described in certain isolated vertigo syndromes affecting the vestibular nucleus (e.g., spontaneous horizontal-torsional nystagmus, abnormal HIT, and ocular tilt reaction), nucleus prepositus hypoglossi (e.g., gaze-evoked nystagmus that is greater ipsilesionally, and ipsilesional spontaneous nystagmus), flocculus (e.g., gaze-evoked nystagmus and abnormal HIT contralesionally, central pattern of head-shaking nystagmus), inferior cerebellar peduncle in the lateral medullary syndrome (e.g., ocular lateropulsion, hypermetric ipsiversive saccades and hypometric contraversive saccades), and nodulus (e.g., periodic alternating nystagmus, central patterns of head-shaking nystagmus). ${ }^{31-33}$

Hearing symptoms, although usually suggestive of a peripheral cause such as labyrinthitis, can be an important clue to a diagnosis of AICA infarction. Acute hearing loss that is ipsilesional to unilateral vestibular loss in the AVS should raise suspicion for labyrinthine ischemia. ${ }^{34}$ By evaluating auditory function with finger rub in addition to performing the HINTS examination (so-called HINTS "Plus"), ${ }^{35}$ the sensitivity to detect an acute stroke increases (HINTS 96.5\% and HINTS
"Plus" 99.1\%) with only a minimal decrease in specificity (HINTS 84.4\%; HINTS "Plus" 83.1\%). ${ }^{34,36}$ Simply put, so long as they are performed in the appropriate circumstance, the HINTS examination differentiates peripheral (vestibular neuritis) from central (brainstem/cerebellar stroke) causes of the AVS, while the HINTS "Plus" examination adds greater sensitivity to detect acute ischemia also involving the labyrinth.

In addition to the HINTS "Plus" examination, head-shaking nystagmus (HSN) can be helpful in differentiating peripheral versus central causes of the AVS. ${ }^{22}$ Following headshaking, it should be noted if nystagmus was provoked or whether spontaneous nystagmus that was present has changed direction. Ideally, fixation should be removed, which can be carried out by performing occlusive ophthalmoscopy (i.e., viewing one optic nerve while covering the fellow eye) or with Frenzel goggles. ${ }^{34,37}$ Central patterns of HSN include vertical nystagmus produced by horizontal head-shaking (known as "perverted" HSN), reversal of nystagmus (e.g., spontaneous right-beating nystagmus at baseline transitioning to left-beating after head-shaking), and vigorous HSN in the absence of unilateral vestibular loss. ${ }^{38,39}$

The etiology of vestibular neuritis is not clear; it may relate to latent herpes simplex virus type 1 in many cases, ${ }^{40}$ although use of antiviral medications has not been found to be effective. ${ }^{41}$ Corticosteroids may hasten clinical recovery 42 or lead to greater improvement in results of vestibular laboratory testing (e.g., calorics), but there are no clear data to suggest that the final clinical outcome is better in those who receive steroids. ${ }^{43}$ Vestibular physical therapy should be recommended to optimize compensation and recovery, ${ }^{42,44,45}$ while vestibular suppressants, such as meclizine, should be discontinued after several days so that normal compensation is not delayed. Fortunately, recurrent or sequential vestibular neuritis (resulting in bilateral vestibular loss) is $\operatorname{rare}^{46}$ (- Table 2 ).

\section{Pearls and Pitfalls}

Recent data indicate that about half of patients presenting with dizziness to the emergency department undergo head CT. ${ }^{47,48}$ However, head CT has a very low sensitivity $(<40 \%)$ for ischemic stroke, ${ }^{49}$ which is the most common central cause of acute dizziness. ${ }^{9}$ For posterior fossa ischemic stroke presenting with dizziness, its sensitivity is estimated to be lower than $10 \% .{ }^{50}$ Therefore, head CT is not useful to rule out stroke in acute dizziness, and often serves only to falsely reassure the ordering clinician.

As opposed to immediately concluding that a vertiginous elderly patient has a stroke or TIA, or that a vertiginous young patient has a peripheral etiology, the examination in the AVS should guide testing, and age should not be overemphasized. ${ }^{27}$ Recent studies suggest that younger patients with dizziness may be at a higher risk of stroke misdiagnosis than older patients. ${ }^{35,51}$

HINTS was specifically evaluated in the context of the acute vestibular syndrome. ${ }^{29}$ It is critical for clinicians to understand that HINTS cannot be applied in episodic vestibular syndromes (EVS). ${ }^{34}$ For example, in migraine, Meniere's or BPPV, the HIT should be normal, and per the HINTS exam, a 
Table 2 Most commonly performed audiovestibular laboratory tests, and the specific conditions in which they may assist in making or supporting the diagnosis

\begin{tabular}{|l|l|l|l|l|l|l|l|l|l|l|l|}
\hline Test & $\begin{array}{l}\text { TIA/ } \\
\text { stroke }\end{array}$ & VN & VM & Meniere's & SCDS & BPPV & VP & BVL & PPPD & $\begin{array}{l}\text { Cerebellar } \\
\text { syndrome }\end{array}$ & $\begin{array}{l}\text { Acoustic } \\
\text { neuroma }\end{array}$ \\
\hline Audiogram & + & + & + & ++ & ++ & - & + & ++ & - & - & ++ \\
\hline vHIT & + & ++ & - & - & - & - & - & ++ & - & + & ++ \\
\hline VNG, ENG or VOG & $+^{\mathrm{a}}$ & $+^{\mathrm{a}}$ & - & - & $+^{\mathrm{b}}$ & $+^{\mathrm{b}}$ & $+^{\mathrm{b}}$ & - & - & - & $++^{\mathrm{b}}$ \\
\hline Rotary chair & - & $+^{\mathrm{c}}$ & $+^{\mathrm{c}}$ & - & - & - & - & ++ & - & - & + \\
\hline Calorics & $+^{\mathrm{d}}$ & ++ & - & + & - & - & - & ++ & - & - & ++ \\
\hline VEMPs & - & $+^{\mathrm{e}}$ & - & + & ++ & - & - & + & - & - & - \\
\hline Imaging $^{\mathrm{f}}$ & ++ & - & + & + & ++ & - & ++ & $+(+)$ & - & ++ & ++ \\
\hline
\end{tabular}

Abbreviations: BPPV, benign paroxysmal positional vertigo; BVL, bilateral vestibular loss; ENG, electronystagmography; PPPD, persistent postural perceptual dizziness (no specific test is helpful to make this diagnosis, but patients often develop PPPD as the result of another poorly treated or anxiety-provoking vestibular disorder, e.g., vestibular migraine, BPPV or vestibular neuritis); VEMPs, vestibular evoked myogenic potentials; vHIT, video head impulse test; VM, vestibular migraine; VN, vestibular neuritis; VNG, video-nystagmography; VOG, videooculography; VP, vestibular paroxysmia; SCDS, superior canal dehiscence syndrome.

Source: Neuro-Ophthalmology Virtual Education Library: NOVEL. [online] Available from: https://collections.lib.utah.edu/ark:/87278/s6zh14pc. Note: $(-)$ Not usually helpful; $(+)$ may be helpful; $(++)$ very helpful.

an the acute setting when spontaneous nystagmus is present.

bDuring provocative maneuvers (including Valsalva, pinched-nose Valsalva, tragal compression, loud sounds, Dix-Hallpike/supine roll test, hyperventilation).

${ }^{\mathrm{c}}$ Rotary chair may show slightly low time constants and gains with unilateral vestibular loss, and may show prolonged time constants in migraine. ${ }^{\mathrm{d}}$ Depending on the posterior fossa localization, there may be caloric weakness with some strokes.

'VEMPs may assist in the localization of inferior versus superior division vestibular neuritis.

${ }^{f}$ Neuroimaging is needed (MRI internal auditory canal [IAC]) protocol w/wo) in migraine and Meniere's cases where there is diagnostic uncertainty or atypical features. In SCDS, CT temporal bones is the test of choice. For vestibular paroxysmia, heavily T2-weighted CISS or FIESTA imaging. In BVL, if the cause is clearly ototoxicity from gentamicin for instance, neuroimaging will not be helpful. Otherwise, neuroimaging is essential.

For additional reading on audiometry: https://collections.lib.utah.edu/details?id=1306738.

For additional reading on vHIT: https://collections.lib.utah.edu/ark:/87278/s62n91vq.

For additional reading on VNG, ENG, and VOG: https://collections.lib.utah.edu/ark:/87278/s6fz193n.

For additional reading on rotary chair: https://collections.lib.utah.edu/ark:/87278/s6b60jbs.

For additional reading on calorics: https://collections.lib.utah.edu/ark:/87278/s6kq21sh.

For additional reading on VEMPs: https://collections.lib.utah.edu/ark:/87278/s66d9smh.

negative HIT would then erroneously suggest a central disorder. Of note, the HINTS examination's sensitivity and specificity were based on examinations conducted within 72 hours of symptom onset in patients with continuous symptoms and spontaneous nystagmus in the AVS. ${ }^{29,34}$

A patient with vestibular neuritis may develop compensatory "covert" saccades during the HIT within days to weeks of the injury. As opposed to "overt" refixation saccades that occur shortly after head movements (this is the catch-up saccade that is visible at the bedside), "covert" saccades occur with head movements and cannot be detected at the bedside. This is clinically relevant since a patient with effective covert saccades may appear to have a normal HIT (see example: https://collections.lib.utah.edu/ ark:/87278/s6r53kbq). The video head impulse test has the advantage of recording both covert and overt saccades, while also calculating a VOR gain based on the ratio of eye movement velocity (output) to head movement velocity (input; - Table 2).

While the HINTS examination is highly effective, its limitations must be understood, particularly the fact that it can only be relied upon to triage and diagnose patients who have AVS. As mentioned above, labyrinthine and brainstem/ cerebellar ischemia can occasionally cause an abnormal HIT. Rarely, an unrecognized congenital fourth nerve palsy (or other vertical strabismus) may lead to the false assumption that an acute skew deviation is present. Also, patients with vestibular neuritis can rarely have vertical diplopia due to a small skew deviation (i.e., a "peripheral skew").

\section{Box 3: Acute Vestibular Syndrome}

\section{Pitfalls}

- HINTS cannot be relied upon in patients with episodic vestibular disorders or in patients with continuous symptoms but without spontaneous nystagmus (e.g., a patient whose symptoms are due to a medical condition).

- In the acute vestibular syndrome, the HINTS examination is more sensitive than neuroimaging, as CT misses approximately $90 \%$ of vestibular strokes, while MRI misses approximately $20 \%$ of vestibular strokes in the first 24 to 48 hours.

- Beware of patients with AICA territory ischemia, who may have peripheral and central vestibular signs, and assess auditory function to evaluate for labyrinthine (cochlear) ischemia.

\section{Pearls}

- HINTS and HINTS "Plus" are superior to MR-DWI in AVS in the first several days. 


\section{Episodic Vestibular Syndrome}

\section{Triggered Episodic Vestibular Syndrome}

\section{Benign Paroxysmal Positional Vertigo}

The characteristic history for benign paroxysmal positional vertigo (BPPV) is $<1$ to 2 minutes of vertigo, which occurs when rolling over in bed, looking up or down, going from the supine to seated position, or the seated to supine position. In PC $\mathrm{BPPV}$, following the DH maneuver, a brief, upbeat-torsional nystagmus (top pole beating toward the lowermost or affected ear) is seen with a latency (a few seconds to occasionally 30 seconds or more) and fatigability on repeated testing. ${ }^{52}$ If a patient has horizontal canal (HC) BPPV, horizontal nystagmus is typically observed with $\mathrm{DH}$, although the supine roll test should also be performed. In this maneuver, the patient's head is simply turned 90 degrees to each side. If cervical range of motion is poor, the patient can be rolled 90 degrees to each side. The Epley or Semont repositioning maneuvers have been shown to be highly effective in treating PC-BPPV (http://content.lib.utah.edu/cdm/ ref/collection/EHSL-NOVEL/id/2350), while the Gufoni (http:// content.lib.utah.edu/cdm/ref/collection/EHSL-NOVEL/id/2351), or BBQ roll (http://content.lib.utah.edu/cdm/ref/collection/ EHSL-NOVEL/id/2352) help treat HC-BPPV. ${ }^{53}$

\section{Pearls and Pitfalls}

If the history is not typical for BPPV, or anything other than the expected pattern of nystagmus is seen, neuroimaging is warranted to exclude a posterior fossa lesion..$^{54}$ Positional downbeat nystagmus is one pattern that may have a central cause, and is normally triggered by DH testing or straighthead hanging (patient is supine and the head is extended 20 to 30 degrees without rotation; - Table 1). Another central pattern is apogeotropic positional nystagmus, which is best triggered by supine roll testing (right-beating nystagmus in left roll and left-beating nystagmus in right roll, i.e., beats away from the earth), but is typically seen with DH as well. The distinction between apogeotropic HC BPPV and central apogeotropic nystagmus can be convoluted (particularly since both can persist for minutes; with HC BPPV, this persistence is due to cupulolithiasis), but the following argue for a peripheral (BPPV) localization: predictable change in nystagmus with bow (head flexed forward), lean (head flexed backward), and supine tests; resolution (or change to geotropic nystagmus) with properly performed repositioning maneuvers (e.g., Gufoni); normal ocular motor and general neurologic (including gait evaluation) examination. The following argue for a central (posterior fossa) localization: unexpected nystagmus vector given a particular positional maneuver; persistence despite repeated and properly performed repositioning maneuvers; additional ocular motor or general neurologic exam abnormalities. ${ }^{55}$ Another important diagnostic consideration is vestibular migraine, as these patients commonly have positional vertigo and nystagmus, which is usually mild to moderate in nystagmus intensity and symptom severity. This is particularly marked during an acute attack. ${ }^{56}$ Commonly, patients with BPPV will have lingering mild dizziness and unsteadiness in between BPPV attacks.

\section{Superior Canal Dehiscence Syndrome}

Superior canal dehiscence syndrome (SCDS) is caused by the creation of a third mobile window in the inner ear, in addition to the oval and round windows, as the result of a dehiscence of the superior (anterior) semicircular canal (i.e., thinning or discontinuity of the bone that separates the superior canal from the middle cranial fossa). ${ }^{26}$ There are believed to be both congenital and acquired factors which may contribute to the development of SCDS. ${ }^{57}$ One large study evaluating the temporal bone in patients with SCDS suggested it is primarily a congenital phenomenon. ${ }^{58} \mathrm{How}$ ever, in about one quarter of cases, another inciting injury such as a traumatic head injury or Valsalva is implicated. ${ }^{59}$

Symptoms suggestive of SCDS include Tullio phenomenon (i.e., provocation of vertigo and nystagmus by loud sounds) and Hennebert sign (i.e., vertigo and nystagmus induced by pressure in the external auditory canal). ${ }^{25,26}$ With sound or pressure triggers, transient symptoms include unsteadiness, vertigo, oscillopsia (due to nystagmus), or rarely an ocular tilt reaction, in which the head is tilted away from the stimulated side and a skew deviation of the eyes develops with the eye higher on the stimulated side. ${ }^{26,60,61}$ Chronic symptoms may include disequilibrium or autophony (perception of internal noises, e.g., hearing one's pulse, eye movements, joints moving/creaking, or one's own footsteps). ${ }^{62}$

The diagnosis of SCDS can be established based on lowered thresholds for eliciting cervical and ocular vestibular evoked myogenic potentials. ${ }^{63-65}$ Characteristic audiometric findings include supranormal bone conduction thresholds with an air-bone gap, reflecting an increased excitability of the labyrinth due to a third mobile window. ${ }^{63}$ One consequence of supranormal bone conduction is that when a tuning fork is placed on the lateral malleolus, it may be heard in the affected ear (i.e., positive malleolus sign). The definitive diagnosis is based on high-resolution $(0.5 \mathrm{~mm}) \mathrm{CT}$ of the temporal bones, demonstrating an abnormal dehiscence $^{66}$ (-Table 2).

Many patients can avoid activities that provoke symptoms and do not require surgery. Refractory cases can be treated with surgical resurfacing and plugging of the superior canal. ${ }^{59}$ Interestingly, SCDS and migraine often coexist, which likely represents the high prevalence of migraine in the general population and SCDS as an effective migraine trigger. ${ }^{57}$ Therefore, adequate control of vestibular migraine is essential to distinguish SCDS from migraine symptoms, so the most appropriate medical or surgical intervention can be selected. Optimal control of vestibular migraine will also minimize the likelihood of a postoperative migraine exacerbation in a patient undergoing SCDS surgery. ${ }^{57}$

\section{Box 4: Episodic Vestibular Syndrome, Triggered Pitfalls}

- Not all forms of positional vertigo and nystagmus is benign; therefore, when atypical features (for BPPV) are 
present (especially when nystagmus is downbeat or apogeotropic), a posterior fossa disorder should be considered.

\section{Pearls}

- Repositioning maneuvers are highly effective in the treatment of BPPV, particularly for posterior and horizontal canal variants.

- If triggers include sound or pressure, consider SCDS.

\section{Spontaneous Episodic Vestibular Syndrome}

\section{Vestibular Migraine}

A diagnosis of vestibular migraine requires the following ${ }^{67}:$ (1) at least five episodes with vestibular symptoms of moderate or severe intensity, lasting 5 minutes to 72 hours; (2) current or previous history of migraine according to the International Classification of Headache Disorders (ICHD); and (3) one or more migraine features with at least $50 \%$ of the vestibular episodes ( $\geq 2$ headache features, photophobia/phonophobia, and visual aura). Although the pathophysiology of vestibular migraine is not entirely understood, it is thought to relate to abnormal interactions between the pain and vestibular pathways, as they converge onto brainstem structures (locus coeruleus, dorsal raphe nucleus, and parabrachial nucleus), leading to overactivation of vestibulothalamocortical pathways. ${ }^{68-72}$ In addition, peripheral mechanisms may contribute as follows: changes in the trigeminovascular system may cause labyrinthine vasodilation, with plasma extravasation and inflammation mediated by the actions of substance $P$, neurokinin $\mathrm{A}$, and calcitonin gene-related peptide (CGRP). ${ }^{70,73-76}$

Hour- to day-long attacks of vertigo are most common, but minutes and even seconds of vertigo can occur in vestibular migraine. ${ }^{68}$ Distinguishing features for vestibular migraine include concurrent or past history of migraine headache (usually with a family history), or at least migrainous features during vertigo attacks (photo/phonophobia, aura, and menstrual precipitation). ${ }^{72}$ Migraine sufferers, especially those with associated vestibular symptoms, are more susceptible to motion sickness. ${ }^{77-79}$ The pathophysiology of motion sickness is thought to involve the vestibular nuclei and a variety of neurotransmitters that are also implicated in migraine. ${ }^{70}$ Histamine, acetylcholine, and noradrenaline are thought to be involved in motion sickness, and their role is supported by the effectiveness of treatment using antihistamines, scopolamine and amphetamines. ${ }^{80-82}$ Serotonin has also been implicated in motion sickness. ${ }^{70}$

Visual vertigo is a condition that may occur subsequent to the development of any vestibular condition, but is quite common in vestibular migraine. Visual vertigo is a specific form of chronic dizziness in which symptoms can be provoked by disorienting, highly patterned visual environments such as crowds, people walking, cars passing by, supermarket aisles, certain geometric patterns, three-dimensional (3D) movies, and large screen televisions. ${ }^{83,84}$ Visual vertigo symptoms are thought to be due to an overreliance on visual cues for posture and orientation. ${ }^{85}$ Such "visual dependence" is thought to develop from a lack of confidence in vestibular or somatosensory inputs that can occur, for example, in patients with vestibular disorders. Optokinetic exposure therapy may be helpful. ${ }^{86,87}$

There is no specific pattern of audiovestibular test findings in vestibular migraine (-Table 2). During attacks of vestibular migraine, spontaneous, headshaking-induced and positional nystagmus are often observed ${ }^{56}$ (- Table 1). Lowvelocity, horizontal positional nystagmus is more common than vertical or torsional nystagmus. The ocular motor and vestibular examination in between attacks is typically normal. After years of vestibular migraine, the examination may show abnormalities such as central positional nystagmus. ${ }^{88}$

Conditions with audiovestibular features and headaches that can mimic vestibular migraine ${ }^{22}$ include Susac syndrome, lupus, antiphospholipid antibody syndrome, Sjogren syndrome, MELAS (mitochondrial encephalopathy, lactic acidosis, and stroke-like episodes), CADASIL (cerebral autosomal dominant arteriopathy with subcortical infarcts and leukoencephalopathy), episodic ataxia, hemiplegic migraine, spinocerebellar atrophy (SCA) 6, and vertebral artery dissection. Each of these conditions has its own unique examination, laboratory, or radiologic findings that allow for the specific diagnosis to be confirmed. MRI with an internal auditory canal protocol should be considered if the history or examination are not typical for migraine, particularly in patients presenting with a first attack of dizziness or vertigo that may or may not represent migraine.

Treatment of vestibular migraine includes dietary and lifestyle modifications, and/or the use of migraine prophylactic medications. Large-scale randomized controlled trials evaluating abortive (e.g., triptans) and preventive medications are lacking, but anecdotally, most of the preventative agents used for migraine headaches are probably effective for vestibular symptoms as well.

\section{Meniere's Disease}

Meniere's disease is characterized by vertigo attacks, lasting 20 minutes to 12 hours, with concurrent hearing loss, tinnitus and aural fullness. ${ }^{89}$ Although the exact cause remains unclear, the associated pathology is thought to be endolymphatic hydrops, which is the expansion of the endolymphatic space within the labyrinth. ${ }^{90-92}$

Initially, vestibular or auditory symptoms can occur in isolation but over time the complete set of symptoms almost always develops. ${ }^{93}$ Spontaneous nystagmus during attacks can vary, depending on which phase of the attack the patient is in (e.g., ipsilesional during the initial excitatory or irritative phase, or contralesional in the subsequent inhibitory phase). Initially, the hearing loss and tinnitus may resolve completely following an attack. Fluctuating sensorineural hearing loss in the low-mid frequency range, documented by audiography, is characteristic (-Table 2). With time, the entire frequency range can progressively become affected in untreated or refractory cases, resulting in severe permanent 
hearing loss. Meniere's disease becomes bilateral in about half of patients. A drop attack termed Tumarkin's otolithic crisis can occur at all stages of Meniere's disease in which patients feel a sudden, severe pushing to the ground by some external force. ${ }^{93}$ This is likely due to an abrupt loss of vestibulospinal reflexes from saccular dysfunction.

The treatment of Meniere's disease mainly consists of sodium restriction and diuretics for mild cases. Intratympanic steroids and gentamicin injection are used in severe refractory cases. ${ }^{94-96}$ Symptomatic relief can be achieved, but no treatment has been clearly shown to halt the progressive destruction of the inner ear in Meniere's disease. ${ }^{97}$

\section{Vestibular Paroxysmia}

Vestibular paroxysmia is thought to result from microvascular compression of the vestibular nerve, analogous to the wellknown pathophysiology of trigeminal neuralgia. ${ }^{22}$ The attacks associated with vestibular paroxysmia are short-lived (most often lasting a few seconds to minutes), tend to occur many times each day, and are often provoked by positional changes or exercise. These attacks represent a diagnostic challenge because clinical evaluation during such a short attack is rare and patients are asymptomatic between attacks. Transient symptoms may include vertigo, oscillopsia, tinnitus, aural fullness, or disequilibrium. ${ }^{98,99}$ Attacks are thought to be related to ephaptic transmission and may be induced by head movement. Hyperventilation-induced nystagmus is seen in approximately $70 \%$ of patients, probably because hyperventilation raises CSF $\mathrm{pH}$ and can transiently increase conductivity across a demyelinated segment of $\mathrm{CN} 8 .{ }^{99}$

A vascular loop that is in contact with CN 8 (usually AICA, vertebral artery, posterior inferior cerebellar artery, or a vein $^{98}$ ) is best seen on heavily T2-weighted sequences such as constructive interference in steady state (CISS) or fast imaging employing steady-state acquisition (FIESTA) imaging. With high resolution CISS or FIESTA imaging, neurovascular contact in the region of the IAC involving CN 8 and/or CN 7 is often observed as a normal finding, raising the conundrum of whether the contact is causal or coincidental (-Table 2). Studies have found that neurovascular contacts between vessels and the eighth cranial nerve can be found in over $20 \%$ of healthy asymptomatic individuals. ${ }^{100}$ In these instances, the history and examination are most important, and trial of an antiseizure medication like carbamazepine or oxcarbazepine can be both diagnostic and therapeutic. ${ }^{99}$ Vestibular testing results consistent with vestibular hypo- or hyperfunction have been described. ${ }^{98}$ Abnormal brainstem auditory-evoked potentials may be another marker for this syndrome.

\section{Box 5: Episodic Vestibular Syndrome, Spontaneous}

\section{Pitfalls}

- Before diagnosing a patient presenting with one or two vertigo attacks with vestibular migraine or Meniere's, first consider dangerous conditions, including dissection causing head/neck pain or hearing loss due to AICA ischemia.

- If the head/neck pain associated with vertigo or dizziness is abrupt in onset, continuous, or severe, do not assume vestibular migraine is the cause; neuroimaging is indicated.

\section{Pearls}

- Mild aural symptoms (without low-mid frequency sensorineural hearing loss typical of Meniere's) are common in vestibular migraine.

- Vascular loops that appear to contact the eighth cranial nerve are common incidental findings with CISS and FIESTA imaging. The diagnosis of vestibular paroxysmia is made based on history, examination, and often following an empirical trial of an antiseizure medication.

\section{Chronic Vestibular Syndrome}

\section{Persistent Postural Perceptual Dizziness}

One of the more common diagnoses made in tertiary balance disorder centers, along with BPPV and vestibular migraine, is persistent postural perceptual dizziness (PPPD). ${ }^{101}$ This disorder, previously known as chronic subjective dizziness or phobic postural vertigo, has been described after a vestibular insult (even with a normal vestibular examination and test results), medical illness, or psychological stress. ${ }^{101-105}$ The pathophysiology of PPPD is not well known, but it is thought to arise from functional changes in postural control, multisensory information processing, and/or cortical integration of spatial orientation. ${ }^{102,106}$

PPPD symptoms are worse when patients are standing or walking, better with sitting, and absent or minimal in the supine position. Its main features include persistent nonvertiginous symptoms lasting 3 or more months, sensitivity to self or environmental motion, and difficulty with precision tasks including reading or computer work. ${ }^{104}$ Complex visual stimuli seem to be particularly troublesome. Although mood disorders such as anxiety and depression often coexist with the vestibular symptoms, PPPD does not represent a primary psychiatric condition, but rather a chronic functional vestibular disorder with characteristic behavioral features. ${ }^{101}$

Certain selective serotonin reuptake inhibitors and serotonin-norepinephrine reuptake inhibitors have been found to be helpful in treating PPPD along with vestibular physical therapy (mainly desensitization or habituation paradigms). Cognitive-behavioral therapy has shown transient benefits without long-lasting effects. ${ }^{102}$

\section{Bilateral Vestibular Loss}

Bilateral vestibular loss (BVL) is a relatively common cause of severe gait imbalance. Generally, unilateral vestibular loss tends to be well compensated, but bilateral loss can be associated with very significant imbalance and visual instability. ${ }^{22,107}$ Gait imbalance from BVL tends to be worse in the dark and on uneven surfaces. Oscillopsia that occurs with 
head motion is almost always present. This type of oscillopsia is particularly noticeable when walking, running, or riding in a car on a bumpy road.

Clinical suspicion of BVL can be confirmed with dynamic visual acuity assessment, quantitative head-impulse test, rotatory chair, and/or caloric testing ${ }^{108}$ ( - Table 2 ). Loss of four or more lines when assessing dynamic visual acuity (see example of how to perform this maneuver-https://collections.lib.utah. edu/ark:/87278/s6tn19w8) and bilaterally positive head impulses are typical findings on examination. ${ }^{108}$ Another suggestive examination finding is instability of the optic nerve during ophthalmoscopy, where the head is oscillated back and forth while the subject is fixating with the fellow eye.

One of the most common causes of BVL is the systemic administration of gentamicin, which is ototoxic, although usually spares auditory function. Gentamicin-induced vestibular toxicity can even occur with only a few days of use. ${ }^{109}$ The risk of vestibular toxicity is higher in renal failure and with concurrent use of another ototoxic medication. Other causes of BVL include advanced bilateral Meniere's disease, sequential vestibular neuritis, meningitis, inflammatory or autoimmune disease (e.g., Cogan's syndrome), superficial siderosis (often with anosmia and cerebellar dysfunction), acute Wernicke's encephalopathy (especially horizontal vestibular function ${ }^{110}$ ), tumors, head trauma, neuropathies, and neurodegenerative processes (e.g., certain spinocerebellar ataxias [SCAs], especially Friedreich's ataxia, SCA3 [Machado-Joseph], and cerebellar ataxia, neuropathy, vestibular areflexia syndrome [CANVAS]). ${ }^{108,111,112}$ However, in more than half of cases of BVL, the etiology remains unknown. ${ }^{111}$

Physical therapy to promote compensation and reliance on visual and proprioceptive systems is the mainstay of treatment. ${ }^{113}$ Vestibular suppressant medications should be avoided. ${ }^{108}$

\section{Acoustic Neuroma}

Acoustic neuromas (or vestibular schwannoma) are rare tumors with an incidence estimated to be between 1 and 2 in 100,000 persons per year. ${ }^{114}$ Acoustic neuromas originate from the nerve sheath-producing Schwann cells which surround the vestibular nerve. Patients with acoustic neuroma typically present with unilateral progressive hearing loss and tinnitus with chronic imbalance due to ipsilateral VOR impairment. Ocular motor examination may reveal ipsilateral gaze-evoked nystagmus and contralateral vestibular nystagmus, a combination known as Burns nystagmus. ${ }^{22}$ In patients with acoustic neuroma, nystagmus is often induced by hyperventilation ( - Table $\mathbf{1}$ ). An audiogram and contrastenhanced MRI with an internal auditory canal protocol should be considered if acoustic neuroma is suspected clinically (-Table 2). Approximately half of all acoustic neuromas are treated by surgery, about a quarter with radiation, and about a quarter are observed. ${ }^{115}$

\section{Cerebellar Diseases}

Disorders affecting the vestibulocerebellum (which includes the flocculus/paraflocculus and uvula/nodulus) are often associated with characteristic ocular motor defi- cits, and patients may seek care due to dizziness, vertigo, unsteadiness or oscillopsia. ${ }^{22}$ Spontaneous downbeat nystagmus (DBN) is often noticed with flocculus/paraflocculus lesions, which in many patients may be due to loss of inhibition of the anterior semicircular canal pathways, resulting from floccular Purkinje cell dysfunction. ${ }^{22}$ Other theories for DBN include up-down asymmetry in cerebellar Purkinje cells, as well as vertical pursuit or otolithic asymmetry. The flocculus/paraflocculus normally improves the neural integrator (gaze-holding) function of brainstem nuclei (nucleus prepositus hypoglossi-medial vestibular nucleus complex horizontally and interstitial nucleus of Cajal vertically), so that a leaky neural integrator will cause gazeevoked nystagmus (GEN). For example, when a patient with a severe cerebellar disorder looks to the right, the gazeholding mechanism will not be able to keep the eyes in eccentric gaze on the target. This results in a slow phase (leftward) drift back to center, with a subsequent rightward fast phase to move the eyes back to the visual target. The nystagmus becomes left-beating when the patient looks to the left, and vertical nystagmus may be present in the vertical gaze as well. Along with GEN, rebound nystagmus is often observed as well. For example, there is right-beating nystagmus in right gaze, and when the patient returns to primary gaze, transient left-beating (rebound) nystagmus can be seen. Saccadic pursuit and saccadic vestibular-ocular reflex suppression (VORS) are also seen due to a decrease in pursuit gain. Pursuit and VORS will be proportionally saccadic, unless the VOR is lost (e.g., bilateral vestibular loss), in which case VORS will look smoother than pursuit because there will be no VOR to suppress. Positional testing to elicit nystagmus can identify patterns diagnostic of cerebellar disease, such as positional DBN in multiple system atrophy or conditions primarily involving the vestibulocerebellum. ${ }^{116,117}$ Hyperventilation and head shaking can also enhance or produce downbeat nystagmus in cerebellar disease. The neurological examination should emphasize the cerebellar exam and gait instability. With subacute onset of cerebellar dysfunction a paraneoplastic process should be considered, especially in elderly patients. With chronic symptoms, one should mainly consider toxic, nutritional, and neurodegenerative conditions.

\section{Oculopalatal Tremor}

Hypertrophic olivary degeneration manifests as oculopalatal tremor, where palatal tremor is synchronous with pendular nystagmus. ${ }^{22,118}$ The most bothersome consequence of oculopalatal tremor is oscillopsia related to the pendular nystagmus, which can be difficult to treat. Oculopalatal tremor develops weeks to months after a structural brainstem or cerebellar lesion affecting the triangle of Guillain-Mollaret, referring to the pathway connecting the dentate nucleus, red nucleus, and inferior olivary nucleus. ${ }^{22,118} \mathrm{~A}$ brainstem hemorrhage (commonly due to a pontine cavernoma) involving the central tegmental tract is the most common responsible lesion. Another syndrome termed progressive ataxia and palatal tremor (PAPT) has also been described; in this presumed neurodegenerative syndrome, a progressive ataxia and 
palatal/oculopalatal tremor develops in patients without history of a structural brainstem or cerebellar lesion. ${ }^{119-121}$

\section{Box 6: Chronic Vestibular Syndrome Pearls}

- Consider the diagnosis of PPPD in a patient who suffered an event that caused dizziness, vertigo or imbalance (especially in someone with pre-existing anxiety or who had a particularly anxiety-provoking response to the inciting event) that is followed by constant daily nonspinning dizziness with a postural component.

- In patients with BVL, a comprehensive neurological examination is helpful in narrowing the differential in the absence of a clear trigger such as gentamicin. For example, if the patient also has cerebellar ocular motor signs (GEN, saccadic pursuit) and severe neuropathy, think about cerebellar ataxia, neuropathy, and vestibular areflexia syndrome (CANVAS).

- A patient with chronic dizziness or imbalance and unilateral auditory dysfunction should undergo a contrast-enhanced MRI with IAC protocol to evaluate for an acoustic neuroma. Rarely, acoustic neuromas present with acute onset of vestibular or auditory symptoms or signs.

- Visualization of the palate (looking for palatal tremor) is important in patients with chronic imbalance/ataxia. OPT can be diagnosed in a patient with pendular nystagmus months to years after a traumatic, hemorrhagic, or ischemic (less common) event. PAPT can be diagnosed in an ataxic patient without nystagmus.

\section{Final Pearls}

As patients with vestibular disorders report symptoms that are often vague and imprecise, a systematic approach to dizziness is essential. Rather than emphasizing symptom quality, it is more helpful to use the Triage-TiTrATE-Test method in order to categorize vestibular disorders based on timing, triggers, and duration, followed by focused oculomotor and vestibular examinations. In the acute vestibular syndrome, clinicians should not overemphasize neuroimaging (head CT is very low yield, and MRI may miss up to $20 \%$ of small posterior fossa strokes in the first 24-48 hours) or general neurologic examination (normal $80 \%$ of the time in patients with isolated dizziness or vertigo due to stroke). Instead, the clinician should focus on the HINTS "Plus" examination, adding head shaking, evaluation of saccades and smooth pursuit, and positional testing (mainly looking for central patterns) as needed. In the spontaneous episodic vestibular syndrome (occurring in isolation or with aural symptoms), clinicians should always consider TIA in the differential, especially in patients with vascular risk factors or head or neck pain suggesting dissection. Vestibular migraine is a much more common disorder compared with Meniere's disease, but when low-mid frequency sensorineural hearing loss is seen in a patient with recurrent vertigo, Meniere's is more likely. In the chronic vestibular syndrome, it is essential to perform a general neurologic examination, including gait assessment, as well as ocular motor and vestibular examinations. Using the systematic approach described in this review, the vast majority of vestibular diagnoses can be readily made at the bedside.

\section{Conflict of Interest}

None.

\section{References}

1 Kerber KA, Morgenstern LB, Meurer WJ, et al. Nystagmus assessments documented by emergency physicians in acute dizziness presentations: a target for decision support? Acad Emerg Med 2011;18(06):619-626

2 Royl G, Ploner CJ, Leithner C. Dizziness in the emergency room: diagnoses and misdiagnoses. Eur Neurol 2011;66(05):256-263

3 Newman-Toker DE, Camargo CA Jr, Hsieh YH, Pelletier AJ, Edlow JA. Disconnect between charted vestibular diagnoses and emergency department management decisions: a cross-sectional analysis from a nationally representative sample. Acad Emerg Med 2009;16(10):970-977

4 Kerber KA, Burke JF, Skolarus LE, et al. Use of BPPV processes in emergency department dizziness presentations: a populationbased study. Otolaryngol Head Neck Surg 2013;148(03):425-430

5 von Brevern M, Radtke A, Lezius F, et al. Epidemiology of benign paroxysmal positional vertigo: a population based study. J Neurol Neurosurg Psychiatry 2007;78(07):710-715

6 Bhattacharyya N, Baugh RF, Orvidas L, et al; American Academy of Otolaryngology-Head and Neck Surgery Foundation. Clinical practice guideline: benign paroxysmal positional vertigo. Otolaryngol Head Neck Surg 2008;139(05, Suppl 4):S47-S81

7 Hilton MP, Pinder DK. The Epley (canalith repositioning) manoeuvre for benign paroxysmal positional vertigo. Cochrane Database Syst Rev 2014;(12):CD003162

8 Hillier SL, McDonnell M. Vestibular rehabilitation for unilateral peripheral vestibular dysfunction. Cochrane Database Syst Rev 2011;(02):CD005397

9 Kerber KA, Meurer WJ, West BT, Fendrick AM. Dizziness presentations in U.S. emergency departments, 1995-2004. Acad Emerg Med 2008;15(08):744-750

10 Jauch EC, Saver JL, Adams HP Jr, et al; American Heart Association Stroke Council; Council on Cardiovascular Nursing; Council on Peripheral Vascular Disease; Council on Clinical Cardiology. Guidelines for the early management of patients with acute ischemic stroke: a guideline for healthcare professionals from the American Heart Association/American Stroke Association. Stroke 2013;44(03):870-947

11 Newman-Toker DE, Cannon LM, Stofferahn ME, Rothman RE, Hsieh YH, Zee DS. Imprecision in patient reports of dizziness symptom quality: a cross-sectional study conducted in an acute care setting. Mayo Clin Proc 2007;82(11):1329-1340

12 Bisdorff A, Von Brevern M, Lempert T, Newman-Toker DE. Classification of vestibular symptoms: towards an international classification of vestibular disorders. J Vestib Res 2009;19(1-2):1-13

13 Moya A, Sutton R, Ammirati F, et al; Task Force for the Diagnosis and Management of Syncope; European Society of Cardiology (ESC); European Heart Rhythm Association (EHRA); Heart Failure Association (HFA); Heart Rhythm Society (HRS). Guidelines for the diagnosis and management of syncope (version 2009). Eur Heart J 2009;30(21):2631-2671 
14 Newman-Toker DE, Edlow JA. TiTrATE: a novel, evidence-based approach to diagnosing acute dizziness and vertigo. Neurol Clin 2015;33(03):577-599, viii

15 Bisdorff AR, Staab JP, Newman-Toker DE. Overview of the international classification of vestibular disorders. Neurol Clin 2015; 33(03):541-550, vii vii

16 Newman-Toker DE. Symptoms and signs of neuro-otologic disorders. Continuum (Minneap Minn) 2012;18(5 Neuro-otology):1016-1040

17 Drachman DA, Hart CW. An approach to the dizzy patient. Neurology 1972;22(04):323-334

18 Stanton VA, Hsieh YH, Camargo CA Jr, et al. Overreliance on symptom quality in diagnosing dizziness: results of a multicenter survey of emergency physicians. Mayo Clin Proc 2007;82(11): 1319-1328

19 Tarnutzer AA, Berkowitz AL, Robinson KA, Hsieh YH, NewmanToker DE. Does my dizzy patient have a stroke? A systematic review of bedside diagnosis in acute vestibular syndrome. CMAJ 2011;183(09):E571-E592

20 Brandt T, Strupp M, Dieterich M. Five keys for diagnosing most vertigo, dizziness, and imbalance syndromes: an expert opinion. J Neurol 2014;261(01):229-231

21 Kerber KA. Acute constant dizziness. Continuum (Minneap Minn) 2012;18(5 Neuro-otology):1041-1059

22 Leigh RJ, Zee DS. The Neurology of Eye Movements. 5th ed. New York: Oxford University Press; 2015

23 Ghavami Y, Mahboubi H, Yau AY, Maducdoc M, Djalilian HR. Migraine features in patients with Meniere's disease. Laryngoscope 2016;126(01):163-168

24 Kaski D, Davies R, Luxon L, Bronstein AM, Rudge P. The Tullio phenomenon: a neurologically neglected presentation. J Neurol 2012;259(01):4-21

25 Minor LB. Superior canal dehiscence syndrome. Am J Otol 2000; 21(01):9-19

26 Minor LB, Solomon D, Zinreich JS, Zee DS. Sound- and/or pressure-induced vertigo due to bone dehiscence of the superior semicircular canal. Arch Otolaryngol Head Neck Surg 1998;124 (03):249-258

27 Kerber KA, Newman-Toker DE. Misdiagnosing dizzy patients: common pitfalls in clinical practice. Neurol Clin 2015;33(03): 565-575, viii viii

28 Paul NL, Simoni M, Rothwell PM; Oxford Vascular Study. Transient isolated brainstem symptoms preceding posterior circulation stroke: a population-based study. Lancet Neurol 2013;12 (01):65-71

29 Kattah JC, Talkad AV, Wang DZ, Hsieh YH, Newman-Toker DE. HINTS to diagnose stroke in the acute vestibular syndrome: three-step bedside oculomotor examination more sensitive than early MRI diffusion-weighted imaging. Stroke 2009;40 (11):3504-3510

30 Newman-Toker DE, Della Santina CC, Blitz AM. Vertigo and hearing loss. Handb Clin Neurol 2016;136:905-921

$31 \mathrm{Kim}$ SH, Park SH, Kim HJ, Kim JS. Isolated central vestibular syndrome. Ann N Y Acad Sci 2015;1343(01):80-89

32 Gold DR, Zee DS. Neuro-ophthalmology and neuro-otology update. J Neurol 2015;262(12):2786-2792

33 Choi JH, Seo JD, Choi YR, et al. Inferior cerebellar peduncular lesion causes a distinct vestibular syndrome. Eur J Neurol 2015; 22(07):1062-1067

34 Kung NH, Van Stavern GP, Gold DR. HINTS in the acute vestibular syndrome: pearls and pitfalls. J Neuroophthalmol 2018;38(02): 244-250

35 Newman-Toker DE, Kerber KA, Hsieh YH, et al. HINTS outperforms $A B C D 2$ to screen for stroke in acute continuous vertigo and dizziness. Acad Emerg Med 2013;20(10):986-996

36 Newman-Toker DE, Curthoys IS, Halmagyi GM. Diagnosing stroke in acute vertigo: the HINTS family of eye movement tests and the future of the "Eye ECG.". Semin Neurol 2015;35(05): 506-521
37 Strupp M, Fischer C, Hanß L, Bayer O. The takeaway Frenzel goggles: a Fresnel-based device. Neurology 2014;83(14): 1241-1245

38 Huh YE, Kim JS. Patterns of spontaneous and head-shaking nystagmus in cerebellar infarction: imaging correlations. Brain 2011;134(Pt 12):3662-3671

39 Choi JY, Jung I, Jung JM, et al. Characteristics and mechanism of perverted head-shaking nystagmus in central lesions: video-oculography analysis. Clin Neurophysiol 2016;127(09):2973-2978

40 Arbusow V, Schulz P, Strupp M, et al. Distribution of herpes simplex virus type 1 in human geniculate and vestibular ganglia: implications for vestibular neuritis. Ann Neurol 1999;46(03): 416-419

41 Strupp M, Zingler VC, Arbusow V, et al. Methylprednisolone, valacyclovir, or the combination for vestibular neuritis. $\mathrm{N}$ Engl J Med 2004;351(04):354-361

42 Goudakos JK, Markou KD, Psillas G, Vital V, Tsaligopoulos M. Corticosteroids and vestibular exercises in vestibular neuritis. Single-blind randomized clinical trial. JAMA Otolaryngol Head Neck Surg 2014;140(05):434-440

43 Fishman JM, Burgess C, Waddell A. Corticosteroids for the treatment of idiopathic acute vestibular dysfunction (vestibular neuritis). Cochrane Database Syst Rev 2011;(05):CD008607

44 Jeong SH, Kim HJ, Kim JS. Vestibular neuritis. Semin Neurol 2013; 33(03):185-194

45 Herdman SJ. Vestibular rehabilitation. Curr Opin Neurol 2013;26 (01):96-101

$46 \mathrm{Kim} \mathrm{YH,} \mathrm{Kim} \mathrm{KS,} \mathrm{Kim} \mathrm{KJ,} \mathrm{Choi} \mathrm{H,} \mathrm{Choi} \mathrm{JS,} \mathrm{Hwang} \mathrm{IK.} \mathrm{Recurrence} \mathrm{of}$ vertigo in patients with vestibular neuritis. Acta Otolaryngol 2011;131(11):1172-1177

47 Kerber KA, Zahuranec DB, Brown DL, et al. Stroke risk after nonstroke emergency department dizziness presentations: a population-based cohort study. Ann Neurol 2014;75(06): 899-907

48 Saber Tehrani AS, Coughlan D, Hsieh YH, et al. Rising annual costs of dizziness presentations to U.S. emergency departments. Acad Emerg Med 2013;20(07):689-696

49 Brazzelli M, Sandercock PA, Chappell FM, et al. Magnetic resonance imaging versus computed tomography for detection of acute vascular lesions in patients presenting with stroke symptoms. Cochrane Database Syst Rev 2009;(04):CD007424

50 Ozono Y, Kitahara T, Fukushima M, et al. Differential diagnosis of vertigo and dizziness in the emergency department. Acta Otolaryngol 2014;134(02):140-145

51 Newman-Toker DE, Moy E, Valente E, Coffey R, Hines AL. Missed diagnosis of stroke in the emergency department: a crosssectional analysis of a large population-based sample. Diagnosis (Berl) 2014;1(02):155-166

52 Kim JS, Zee DS. Clinical practice. Benign paroxysmal positional vertigo. N Engl J Med 2014;370(12):1138-1147

53 Gold DR, Morris L, Kheradmand A, Schubert MC. Repositioning maneuvers for benign paroxysmal positional vertigo. Curr Treat Options Neurol 2014;16(08):307

54 Choi JY, Kim JH, Kim HJ, Glasauer S, Kim JS. Central paroxysmal positional nystagmus: Characteristics and possible mechanisms. Neurology 2015;84(22):2238-2246

55 Choi JY, Glasauer S, Kim JH, Zee DS, Kim JS. Characteristics and mechanism of apogeotropic central positional nystagmus. Brain 2018;141(03):762-775

56 Polensek SH, Tusa RJ. Nystagmus during attacks of vestibular migraine: an aid in diagnosis. Audiol Neurotol 2010;15(04): 241-246

57 Ward BK, Carey JP, Minor LB. Superior canal dehiscence syndrome: lessons from the first 20 years. Front Neurol 2017;8:177

58 Carey JP, Minor LB, Nager GT. Dehiscence or thinning of bone overlying the superior semicircular canal in a temporal bone survey. Arch Otolaryngol Head Neck Surg 2000;126(02): 137-147 
59 Minor LB. Clinical manifestations of superior semicircular canal dehiscence. Laryngoscope 2005;115(10):1717-1727

60 Deecke L, Mergner T, Plester D. Tullio phenomenon with torsion of the eyes and subjective tilt of the visual surround. Ann N Y Acad Sci 1981;374:650-655

61 Dieterich M, Brandt T, Fries W. Otolith function in man. Results from a case of otolith Tullio phenomenon. Brain 1989;112(Pt 5):1377-1392

62 Chien WW, Carey JP, Minor LB. Canal dehiscence. Curr Opin Neurol 2011;24(01):25-31

63 Streubel SO, Cremer PD, Carey JP, Weg N, Minor LB. Vestibularevoked myogenic potentials in the diagnosis of superior canal dehiscence syndrome. Acta Otolaryngol Suppl 2001;545:41-49

64 Zuniga MG, Janky KL, Nguyen KD, Welgampola MS, Carey JP. Ocular versus cervical VEMPs in the diagnosis of superior semicircular canal dehiscence syndrome. Otol Neurotol 2013; 34(01):121-126

65 Rosengren SM, Kingma H. New perspectives on vestibular evoked myogenic potentials. Curr Opin Neurol 2013;26(01):74-80

66 Belden CJ, Weg N, Minor LB, Zinreich SJ. CT evaluation of bone dehiscence of the superior semicircular canal as a cause of sound- and/or pressure-induced vertigo. Radiology 2003;226 (02):337-343

67 Lempert T, Olesen J, Furman J, et al. Vestibular migraine: diagnostic criteria. JVestib Res 2012;22(04):167-172

68 Furman JM, Marcus DA, Balaban CD. Migrainous vertigo: development of a pathogenetic model and structured diagnostic interview. Curr Opin Neurol 2003;16(01):5-13

69 Furman JM, Balaban CD, Jacob RG, Marcus DA. Migraine-anxiety related dizziness (MARD): a new disorder? J Neurol Neurosurg Psychiatry 2005;76(01):1-8

70 Furman JM, Marcus DA. Migraine and motion sensitivity. Continuum (Minneap Minn) 2012;18(5 Neuro-otology):1102-1117

71 Furman JM, Marcus DA, Balaban CD. Vestibular migraine: clinical aspects and pathophysiology. Lancet Neurol 2013;12(07): 706-715

72 Furman JM, Balaban CD. Vestibular migraine. Ann N Y Acad Sci 2015;1343:90-96

73 Vass Z, Shore SE, Nuttall AL, Miller JM. Direct evidence of trigeminal innervation of the cochlear blood vessels. Neuroscience 1998;84(02):559-567

74 Scarfone E, Ulfendahl M, Lundeberg T. The cellular localization of the neuropeptides substance $\mathrm{P}$, neurokinin A, calcitonin generelated peptide and neuropeptide $\mathrm{Y}$ in guinea-pig vestibular sensory organs: a high-resolution confocal microscopy study. Neuroscience 1996;75(02):587-600

75 Kong WJ, Scholtz AW, Kammen-Jolly K, et al. Ultrastructural evaluation of calcitonin gene-related peptide immunoreactivity in the human cochlea and vestibular endorgans. Eur J Neurosci 2002;15(03):487-497

76 Ho TW, Edvinsson L, Goadsby PJ. CGRP and its receptors provide new insights into migraine pathophysiology. Nat Rev Neurol 2010;6(10):573-582

77 Cuomo-Granston A, Drummond PD. Migraine and motion sickness: what is the link? Prog Neurobiol 2010;91(04):300-312

78 Drummond PD. Triggers of motion sickness in migraine sufferers. Headache 2005;45(06):653-656

79 Jeong SH, Oh SY, Kim HJ, Koo JW, Kim JS. Vestibular dysfunction in migraine: effects of associated vertigo and motion sickness. J Neurol 2010;257(06):905-912

80 Takeda N, Morita M, Horii A, Nishiike S, Kitahara T, Uno A. Neural mechanisms of motion sickness. J Med Invest 2001;48(1-2):44-59

81 Shupak A, Gordon CR. Motion sickness: advances in pathogenesis, prediction, prevention, and treatment. Aviat Space Environ Med 2006;77(12):1213-1223

82 Eisenman LM. Motion sickness may be caused by a neurohumoral action of acetylcholine. Med Hypotheses 2009;73(05): 790-793
83 Bronstein AM. Visual vertigo syndrome: clinical and posturography findings. JNeurol Neurosurg Psychiatry 1995;59(05): 472-476

84 Balaban CD, Jacob RG. Background and history of the interface between anxiety and vertigo. J Anxiety Disord 2001;15(1-2):27-51

85 Maire R, Mallinson A, Ceyte $\mathrm{H}$, et al. Discussion about visual dependence in balance control: european society for clinical evaluation of balance disorders. J Int Adv Otol 2017;13(03): 404-406

86 Pavlou M. The use of optokinetic stimulation in vestibular rehabilitation. J Neurol Phys Ther 2010;34(02):105-110

87 Pavlou M, Bronstein AM, Davies RA. Randomized trial of supervised versus unsupervised optokinetic exercise in persons with peripheral vestibular disorders. Neurorehabil Neural Repair 2013;27(03):208-218

88 Radtke A, von Brevern M, Neuhauser H, Hottenrott T, Lempert T. Vestibular migraine: long-term follow-up of clinical symptoms and vestibulo-cochlear findings. Neurology 2012;79(15):1607-1614

89 Lopez-Escamez JA, Carey J, Chung WH, et al; Classification Committee of the Barany Society; Japan Society for Equilibrium Research; European Academy of Otology and Neurotology (EAONO); Equilibrium Committee of the American Academy of Otolaryngology-Head and Neck Surgery (AAO-HNS); Korean Balance Society. Diagnostic criteria for Menière's disease.J Vestib Res 2015;25(01):1-7

90 Rauch SD, Merchant SN, Thedinger BA. Meniere's syndrome and endolymphatic hydrops. Double-blind temporal bone study. Ann Otol Rhinol Laryngol 1989;98(11):873-883

91 Takeda T, Taguchi D. Aquaporins as potential drug targets for Meniere's disease and its related diseases. Handb Exp Pharmacol 2009;(190):171-184

92 Semaan MT, Megerian CA. Contemporary perspectives on the pathophysiology of Meniere's disease: implications for treatment. Curr Opin Otolaryngol Head Neck Surg 2010;18(05): 392-398

93 Huppert D, Strupp M, Brandt T. Long-term course of Menière's disease revisited. Acta Otolaryngol 2010;130(06):644-651

94 Thirlwall AS, Kundu S. Diuretics for Ménière's disease or syndrome. Cochrane Database Syst Rev 2006;(03):CD003599

95 Pullens B, van Benthem PP. Intratympanic gentamicin for Ménière's disease or syndrome. Cochrane Database Syst Rev 2011;(03):CD008234

96 Phillips JS, Westerberg B. Intratympanic steroids for Ménière's disease or syndrome. Cochrane Database Syst Rev 2011;(07): CD008514

97 Lempert T. Recurrent spontaneous attacks of dizziness. Continuum (Minneap Minn) 2012;18(5 Neuro-otology):1086-1101

98 Best C, Gawehn J, Krämer HH, et al. MRI and neurophysiology in vestibular paroxysmia: contradiction and correlation. JNeurol Neurosurg Psychiatry 2013;84(12):1349-1356

99 Hüfner K, Barresi D, Glaser M, et al. Vestibular paroxysmia: diagnostic features and medical treatment. Neurology 2008;71 (13):1006-1014

100 De Carpentier J, Lynch N, Fisher A, Hughes D, Willatt D. MR imaged neurovascular relationships at the cerebellopontine angle. Clin Otolaryngol Allied Sci 1996;21(04):312-316

101 Brandt T. Phobic postural vertigo. Neurology 1996;46(06): 1515-1519

102 Staab JP. Chronic subjective dizziness. Continuum (Minneap Minn) 2012;18(5 Neuro-otology):1118-1141

103 Ruckenstein MJ, Staab JP. Chronic subjective dizziness. Otolaryngol Clin North Am 2009;42(01):71-77, ix ix

104 Staab JP, Ruckenstein MJ. Expanding the differential diagnosis of chronic dizziness. Arch Otolaryngol Head Neck Surg 2007;133 (02):170-176

105 Meurer WJ, Low PA, Staab JP. Medical and psychiatric causes of episodic vestibular symptoms. Neurol Clin 2015;33(03): 643-659, ix ix 
106 Staab JP, Eckhardt-Henn A, Horii A, et al. Diagnostic criteria for persistent postural-perceptual dizziness (PPPD): Consensus document of the committee for the Classification of Vestibular Disorders of the Bárány Society. J Vestib Res 2017;27(04):191-208

107 Living without a balancing mechanism. N Engl J Med 1952;246 (12):458-460

108 Hain TC, Cherchi M, Yacovino DA. Bilateral vestibular loss. Semin Neurol 2013;33(03):195-203

109 Ahmed RM, Hannigan IP, MacDougall HG, Chan RC, Halmagyi GM. Gentamicin ototoxicity: a 23-year selected case series of 103 patients. Med J Aust 2012;196(11):701-704

110 Kattah JC, Dhanani SS, Pula JH, Mantokoudis G, Tehrani ASS, Toker DEN. Vestibular signs of thiamine deficiency during the early phase of suspected Wernicke encephalopathy. Neurol Clin Pract 2013;3(06):460-468

111 Zingler VC, Weintz E, Jahn K, et al. Causative factors, epidemiology, and follow-up of bilateral vestibulopathy. Ann N Y Acad Sci 2009;1164:505-508

112 Rinne T, Bronstein AM, Rudge P, Gresty MA, Luxon LM. Bilateral loss of vestibular function: clinical findings in 53 patients. J Neurol 1998;245(6,7):314-321

113 Porciuncula F, Johnson CC, Glickman LB. The effect of vestibular rehabilitation on adults with bilateral vestibular hypofunction: a systematic review. J Vestib Res 2012;22(5-6):283-298
114 Tos M, Stangerup SE, Cayé-Thomasen P, Tos T, Thomsen J. What is the real incidence of vestibular schwannoma? Arch Otolaryngol Head Neck Surg 2004;130(02):216-220

115 Carlson ML, Habermann EB, Wagie AE, et al. The changing landscape of vestibular schwannoma management in the United States-a shift toward conservatism. Otolaryngol Head Neck Surg 2015;153(03):440-446; [Epub ahead of print]

116 Hüfner K, Stephan T, Kalla R, et al. Structural and functional MRIs disclose cerebellar pathologies in idiopathic downbeat nystagmus. Neurology 2007;69(11):1128-1135

117 Kalla R, Deutschlander A, Hufner K, et al. Detection of floccular hypometabolism in downbeat nystagmus by fMRI. Neurology 2006;66(02):281-283

118 Tilikete C, Desestret V. Hypertrophic olivary degeneration and palatal or oculopalatal tremor. Front Neurol 2017;8:302

119 Papachatzaki MM, Ali N, Arshad Q, et al. Progressive ataxia with oculo-palatal tremor and optic atrophy. J Neurol 2013;260(11): 2903-2905

120 Samuel M, Torun N, Tuite PJ, Sharpe JA, Lang AE. Progressive ataxia and palatal tremor (PAPT): clinical and MRI assessment with review of palatal tremors. Brain 2004;127(Pt 6):1252-1268

121 Gao AF, Faust-Socher A, Al-Murshed M, Del Bigio MR, Lang AE, Munoz DG. Progressive ataxia and palatal tremor: Two autopsy cases of a novel tauopathy. Mov Disord 2017;32(10):1465-1473 